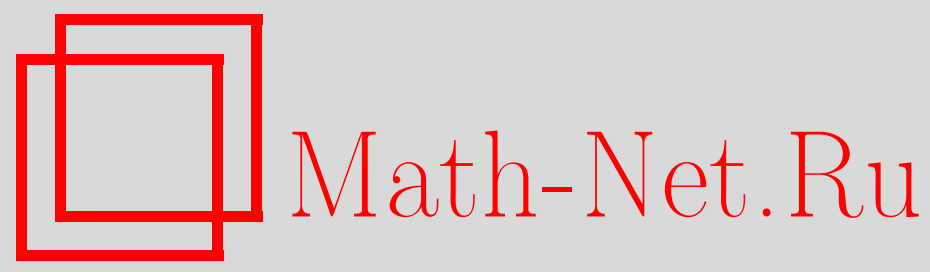

А. Аскари-Хеммат, М. А. Дегхан, М. А. Скопина, Полиномиальные всплескоподобные разложения функций на сфере, Матем. заметки, 2003, том 74, выпуск 2, 292-300

DOI: https://doi.org/10.4213/mzm259

Использование Общероссийского математического портала Math-Net.Ru подразумевает, что вы прочитали и согласны с пользовательским соглашением http://www.mathnet.ru/rus/agreement

Параметры загрузки:

IP: 54.197 .130 .99

26 апреля 2023 г., 13:08:48

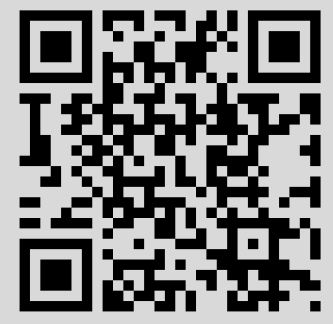




\title{
ПОЛИНОМИАЛЬНЫЕ ВСПЛЕСКОПОДОБНЫЕ РАЗЛОЖЕНИЯ ФУНКЦИЙ НА СФЕРЕ
}

\author{
А. Аскари-Хеммат, М. А. Дегхан, М. А. Скопина
}

\begin{abstract}
Представляются всплескоподобные системы на $S^{d}$ такие, что любая непрерывная функция раскладьвается в ряд по таким "всплескам". Порядок роста степеней полиномов является оптимальным. Коэффициенты разложений являются скалярньми произведениями функции на элементы “двойственной системы всплесков". "Двойственные всплески" также представляют собой полиномиальную систему с тем же ростом степеней полиномов. Система является избыточной. Приводится также конструкция некоторого полиномиального базиса, но в отличие от всплескоподобной системы этот базис непригоден для использования, поскольку, во-первых, нет явньх формул для вычисления коэффициентов, во-вторых, степени полиномов растут слишком быстро.

Библиограф̆ия: 15 названий.
\end{abstract}

1. В одномерном случае полиномиальные разложения непрерывных функций на окружности и на отрезке активно изучались многими математиками на протяжении почти сорока лет. Первьй полиномиальный базис в $C[a, b]$ был найден в 1961 г. Фоишем и Зингером [1]. Рост степеней полиномов в этом базисе экспоненциальный. В 1987 г. Привалов [2] построил полиномиальные базисы в пространстве непрерьвных функций с оптимальным ростом степеней полиномов как для тригонометрического, так и для алгебраического случаев. Однако эти базисы не были ортогональными. Оптимальные тригонометрические полиномиальные ортогональные базисы были найдены в 1994 благодаря развитию теории всплесков. Лоренц и Саакян [3] доказали, что пакеты периодических всплесков Мейера являются такими базисами. Используя некоторые обобщенные операторы сдвигов, третий автор нашел [4] аналогичную всплесковую конструкцию в $L_{2}[a, b]$ и доказал, что соответствующие пакеты всплесков являются оптимальными полиномиальными ортогональными базисами в пространстве $C[a, b]$. Хотя эта конструкция может быть реализована в любом гильбертовом пространстве с полиномиальным базисом, в общем случае не ясно, будут ли ограниченными функции Лебега частичных сумм ряда Фурье по таким всплескам. В частности, очень сомнительно, что такая система будет базисом в $C\left(S^{d}\right)$.

Всплескоподобные полиномиальные системы на двумерной сфере были предложены Фриденом и Шрайнером [5]. Фарков [6] распространил их конструкцию на многомерный

Работа выполнена при поддержке Университета Валиаср, Рафсанжан, Иран, грант № 00-8131-01. Работа третьего автора поддержана Российским фондом фундаментальных исследований, грант № 03-01-00373. 
случай. В отличие от одномерных базисов всплесков эти системы не являются ортогональными. Более того, они не являются базисами в $L_{2}$, и разложение произвольной функции, вообще говоря, не сходится в $L_{2}$. Тем не менее, разложения по таким системам имеют значительное сходство с обычньпи системами всплесков. В частности, в определенном смысле сохраняется структура кратномасштабного анализа. В настоящей статье мы показываем, что для некоторых “сферических всплесков" разложения по ним произвольной функции $f \in C\left(S^{d}\right)$ равномерно сходятся к этой функции. Рост степеней полиномов в этих разложениях является оптимальным с точностью до константы. Для случая $d=2$ такие системы были найдены третьим автором [7].

Наши "всплески" не образуют базиса, система является избыточной. Полиномиальный базис в $C\left(S^{d}\right)$ несложно построить из произвольного базиса в $C\left(S^{d}\right)$ с помощю общего метода, разработанного Фоишем и Зингером [1]. Мы приводим такую конструкцию. $\mathrm{K}$ сожалению, этот базис не пригоден для использования ввиду двух его недостатков: нет явньх формул для коэффициентов разложения, степени полиномов растут слишком быстро.

2. Следующие обозначения будут использоваться во всей статье: $x \cdot y=x_{1} y_{1}+\cdots+$ $x_{d} y_{d},|x|=\sqrt{x \cdot x}$ для $x, y \in \mathbb{R}^{d}, \pi_{n}^{d}$ - пространство полиномов $d$ переменных степени не вьше $n, G_{n}^{(\lambda)}$ - полином Гегенбауэра степени $n$ порядка $\lambda, B^{d}=\left\{x \in \mathbb{R}^{d}:|x| \leqslant 1\right\}$, $S^{d}=\partial B^{d+1}$

$$
\omega_{d}:=\int_{S^{d}} d s(x)=\frac{2 \pi^{(d+1) / 2}}{\Gamma((d+1) / 2)},
$$

скалярное произведение функций $F, G \in L_{2}\left(S^{d}\right)$ будем обозначать

$$
\langle F, G\rangle=\int_{S^{d}} F(x) \overline{G(x)} d s(x) .
$$

Для фиксированного целого $n$ сужение однородного гармонического полинома степени $n$ на $S^{d}$ назьвается сферической гармоникой степени $n$. Следующую информацию можно найти в [8] и $[9$, гл. IV,$\S 2]$. Пусть $H_{n}^{d}$ обозначает класс сферических гармоник степени $n$. Пространства $H_{n}^{d}$ попарно ортогональны, и $\bigoplus_{\ell=0}^{n} H_{\ell}^{d}$ состоит из сужений на $S^{d}$ всех алгебраических полиномов $d+1$ переменной степени не вьше $n$. Пространство $H_{n}^{d}$ имеет размерность

$$
\delta_{n}^{d}:=\operatorname{dim} H_{n}^{d}= \begin{cases}\frac{2 n+d-1}{n+d-1}\left(\begin{array}{c}
n+d-1 \\
n
\end{array}\right), & \text { если } n \geqslant 1, \\
1, & \text { если } n=0 .\end{cases}
$$

Кроме того, $\bigoplus_{n=0}^{\infty} H_{n}^{d}$ плотно в $L_{2}\left(S^{d}\right)$. Следовательно, если мы выберем ортонормированный базис $\left\{Y_{n k}, k=1, \ldots, \delta_{n}^{d}\right\}$ для каждого $H_{n}^{d}$, то функции $\left\{Y_{n k}, n=0,1, \ldots\right.$, $\left.k=1, \ldots, \delta_{n}^{d}\right\}$ образуют ортонормированньй базис в $L_{2}\left(S^{d}\right)$. Имеет место формула суммирования [8]:

$$
\sum_{k=1}^{\delta_{n}^{d}} Y_{n k}(x) Y_{n k}(y)=\frac{(2 n+d-1)}{\omega_{d}(d-1)} G_{n}^{((d-1) / 2)}(x \cdot y)
$$

для всех $x, y \in S^{d}$ и для всех $n=0,1, \ldots$.

Следующее утверждение резюмирует результаты, данные в [10] (см. также [11, теорема 2.1]). 
Теорема 1. Существуют постоянные $N_{d}$ u $A_{d}$, зависящие только от $d$, такие, что для любого множества $\left\{\eta_{\ell}\right\}_{\ell \in \Omega}$, состоящего из различных точек $\eta_{\ell} \in S^{d}, u$ для любого натурального $N \geqslant N_{d}$, удовлетворяющих условию

$$
N \max _{x \in S^{d}} \min _{\ell \in \Omega}\left|x-\eta_{\ell}\right| \leqslant A_{d},
$$

существуют такие неотрицательные веса $a_{\ell}, \ell \in \Omega$, что

$$
\int_{S^{d}} P(x) d s(x)=\sum_{\ell \in \Omega} a_{\ell} P\left(\eta_{\ell}\right)
$$

для всех $P \in \pi_{N}^{d+1}$.

По этой теореме каждому натуральному $j$ можно сопоставить множество $\left\{\eta_{\ell}^{(j)}\right\}_{\ell \in \Omega_{j}}$ различных точек $\eta_{\ell}^{(j)} \in S^{d}$ и множество $\left\{a_{\ell}^{(j)}\right\}_{\ell \in \Omega_{j}}$ неотрицательных весов, обладающих следующими свойствами: $\operatorname{card} \Omega_{j} \sim 2^{d j}$,

$$
\int_{S^{d}} P(x) d s(x)=\sum_{\ell \in \Omega_{j}} a_{\ell}^{(j)} P\left(\eta_{\ell}^{(j)}\right)
$$

для любого $P \in \pi_{2^{j}}^{d+1}$. Введем дополнительно множество $\Omega_{0}:=\{0\}$ и положим $a_{0}^{(0)}=\omega_{d}$

3. Пусть

$$
h_{j}(n)=\frac{\left(\begin{array}{c}
2^{j}-n+d-1 \\
d
\end{array}\right)}{\left(\begin{array}{c}
2^{j}+d-1 \\
2^{j}-1
\end{array}\right)}
$$

для $n=0, \ldots, 2^{j}-1$ и $h_{j}(n)=0$ для $n=2^{j}, 2^{j}+1, \ldots$ Нетрудно узнать в этих величинах множители $(C, d)$-средних порядка $2^{j}-1$. Хорошо известно (см., например, [12]), что ряды Фурье-Гегенбауэра суммируемы методом $(C, d)$ в $C[-1,1]$ при $\lambda<d+1 / 2$. Более того, имеет место неравенство Когбетлянца [13, с. 71]:

$$
\sum_{n=0}^{N}\left(\begin{array}{c}
N-n+d \\
d
\end{array}\right)(2 n+d-1) G_{n}^{((d-1) / 2)}(t) \geqslant 0
$$

для всех $t \in[-1,1]$. Положим $g_{j}(n)=h_{j}(n)+h_{j-1}(n), \widetilde{g}_{j}(n)=h_{j}(n)-h_{j-1}(n)$ для $j=1,2, \ldots, n=0,1, \ldots ; g_{0}(0)=h_{0}(0)+1, \widetilde{g}_{0}(0)=h_{0}(0)-1 ; g_{0}(n)=\widetilde{g}_{0}(n)=0$ для $n=1,2, \ldots$. Для каждого неотрицательного целого $j$ и для любого $\ell \in \Omega_{j+1}$ определим функцию всплесков $\Psi_{j \ell}$, двойственную функцию всплесков $\widetilde{\Psi}_{j \ell}$ и масштабирующую функцию $\Phi_{(j+1) \ell}$ по формулам

$$
\begin{aligned}
\Psi_{j \ell}(x) & =\frac{1}{\omega_{d}(d-1)} \sum_{n \in \mathbb{Z}_{+}} g_{j}(n)(2 n+d-1) G_{n}^{((d-1) / 2)}\left(\eta_{\ell}^{(j+1)} \cdot x\right), \\
\widetilde{\Psi}_{j \ell}(x) & =\frac{1}{\omega_{d}(d-1)} \sum_{n \in \mathbb{Z}_{+}} \widetilde{g}_{j}(n)(2 n+d-1) G_{n}^{((d-1) / 2)}\left(\eta_{\ell}^{(j+1)} \cdot x\right), \\
\Phi_{(j+1) \ell}(x) & =\frac{1}{\omega_{d}(d-1)} \sum_{n \in \mathbb{Z}_{+}} h_{j}(n)(2 n+d-1) G_{n}^{((d-1) / 2)}\left(\eta_{\ell}^{(j+1)} \cdot x\right) .
\end{aligned}
$$


Дополним этот набор функцией $\Phi_{0,0} \equiv 1 / \omega_{d}$ и положим $\Phi_{0}=\sqrt{\omega_{d}} \Phi_{0,0}$.

Для $F \in C\left(S^{d}\right)$ мы будем изучать сходимость ряда

$$
\left\langle F, \Phi_{0}\right\rangle \Phi_{0}+\sum_{i=0}^{\infty} \sum_{\ell \in \Omega_{i+1}} a_{\ell}^{(j+1)}\left\langle F, \widetilde{\Psi}_{i \ell}\right\rangle \Psi_{i \ell}
$$

Положим

$$
\Lambda_{j, \omega}(F)=\left\langle F, \Phi_{0}\right\rangle \Phi_{0}+\sum_{i=0}^{j-1} \sum_{\ell \in \Omega_{i+1}} a_{\ell}^{(j+1)}\left\langle F, \widetilde{\Psi}_{i \ell}\right\rangle \Psi_{i \ell}+\sum_{\ell \in \omega} a_{\ell}^{(j+1)}\left\langle F, \widetilde{\Psi}_{j \ell}\right\rangle \Psi_{j \ell},
$$

где $\omega$ - подмножество множества $\Omega_{j+1}$.

ЛЕмма 2. Для любого $F \in C\left(S^{d}\right)$

$$
\left\langle F, \Phi_{0}\right\rangle \Phi_{0}+\sum_{i=0}^{j-1} \sum_{\ell \in \Omega_{i+1}} a_{\ell}^{(j+1)}\left\langle F, \widetilde{\Psi}_{i \ell}\right\rangle \Psi_{i \ell}=\sum_{\ell \in \Omega_{j}} a_{\ell}^{(j)}\left\langle F, \Phi_{j \ell}\right\rangle \Phi_{j \ell} .
$$

ДокАЗАТЕЛЬСТво. На основании (2) имеем

$$
\begin{aligned}
& \sum_{\ell \in \Omega_{j}} a_{\ell}^{(j)} \Phi_{j \ell}(t) \Phi_{j \ell}(x)=\sum_{n \in \mathbb{Z}_{+}} \sum_{k \in \mathbb{Z}_{+}} h_{j-1}(n) h_{j-1}(k) \\
& \quad \times \int_{S^{d}} d s(\eta) \frac{(2 n+d-1)}{\omega_{d}(d-1)} G_{n}^{((d-1) / 2)}(\eta \cdot t) \frac{(2 k+d-1)}{\omega_{d}(d-1)} G_{k}^{((d-1) / 2)}(\eta \cdot x) .
\end{aligned}
$$

Отсюда, используя (1) и принимая во внимание ортонормированность системы $\left\{Y_{n k}\right\}_{n, k}$, имеем

$$
\begin{aligned}
\sum_{\ell \in \Omega_{j}} a_{\ell}^{(j)}\left\langle F, \Phi_{j \ell}\right\rangle \Phi_{j \ell}(x)= & \int_{S^{d}} d s(t) F(t) \sum_{n \in \mathbb{Z}_{+}} \sum_{k \in \mathbb{Z}_{+}} h_{j-1}(n) h_{j-1}(k) \\
& \times \int_{S^{d}} d s(\eta) \sum_{\nu=1}^{\delta_{n}^{d}} Y_{n \nu}(\eta) Y_{n \nu}(t) \sum_{\kappa=1}^{\delta_{k}^{d}} Y_{k \kappa}(\eta) Y_{k \kappa}(x) \\
= & \int_{S^{d}} F(t) \sum_{n \in \mathbb{Z}_{+}} h_{j-1}^{2}(n) \sum_{\nu=1}^{\delta_{n}^{d}} Y_{n \nu}(x) Y_{n \nu}(t) d s(t) .
\end{aligned}
$$

Аналогично,

$$
\sum_{\ell \in \Omega_{i+1}} a_{\ell}^{(i+1)}\left\langle F, \widetilde{\Psi}_{i \ell}\right\rangle \Psi_{i \ell}(x)=\int_{S^{d}} F(t) \sum_{n \in \mathbb{Z}_{+}} \widetilde{g}_{i}(n) g_{i}(n) \sum_{\nu=1}^{\delta_{n}^{d}} Y_{n \nu}(x) Y_{n \nu}(t) d s(t) .
$$

Поскольку $\widetilde{g}_{i}(n) g_{i}(n)=h_{i}^{2}(n)-h_{i-1}^{2}(n)$, отсюда следует

$$
\sum_{\ell \in \Omega_{i}} a_{\ell}^{(i)}\left\langle F, \Phi_{i \ell}\right\rangle \Phi_{i \ell}=\sum_{\ell \in \Omega_{i-1}} a_{\ell}^{(i-1)}\left\langle F, \Phi_{(i-1) \ell}\right\rangle \Phi_{(i-1) \ell}+\sum_{\ell \in \Omega_{i}} a_{\ell}^{(i)}\left\langle F, \widetilde{\Psi}_{(i-1) \ell}\right\rangle \Psi_{(i-1) \ell} .
$$

Просуммировав эти равенства по всем $i=1, \ldots, j$ получаем (4). 
Tеорема 3. Для любого $F \in C\left(S^{d}\right)$

$$
\lim _{j \rightarrow \infty}\left\|F-\Lambda_{j, \omega}(F)\right\|_{\infty}=0
$$

причем сходимость равномерная относительно выбора $\omega \subset \Omega_{j+1}$.

ДоказАтЕЛЬСтво. Сначала мы докажем, что операторы $\Lambda_{j, \omega}$, действующие из $C\left(S^{d}\right)$ в $C\left(S^{d}\right)$, равномерно ограничены. По (4), (2) и (3)

$$
\begin{aligned}
&\left|\Lambda_{j, \varnothing}(F, x)\right|=\left|\sum_{\ell \in \Omega_{j}} a_{\ell}^{(j)}\left\langle F, \Phi_{j \ell}\right\rangle \Phi_{j \ell}\right| \\
&=\mid \int_{S^{d}} d s(\eta) \sum_{k \in \mathbb{Z}_{+}} h_{j-1}(k) \frac{(2 k+d-1)}{\omega_{d}(d-1)} G_{k}^{((d-1) / 2)}(\eta \cdot x) \\
& \quad \times \int_{S^{d}} d s(t) F(t) \sum_{n \in \mathbb{Z}_{+}} h_{j-1}(n) \frac{(2 n+d-1)}{\omega_{d}(d-1)} G_{n}^{((d-1) / 2)}(\eta \cdot t) \mid \\
& \leqslant\|F\|_{\infty} \int_{S^{d}} d s(\eta) \sum_{k \in \mathbb{Z}_{+}} h_{j-1}(k) \frac{(2 n+d-1)}{\omega_{d}(d-1)} G_{n}^{((d-1) / 2)}(\eta \cdot x) \\
& \times \int_{S^{d}} d s(t) \sum_{n \in \mathbb{Z}_{+}} h_{j-1}(n) \frac{(2 n+d-1)}{\omega_{d}(d-1)} G_{n}^{((d-1) / 2)}(\eta \cdot t) .
\end{aligned}
$$

Поскольку, ввиду ортонормированности системы $\left\{Y_{n k}\right\}_{n, k}$

$$
Y_{01} \equiv \frac{1}{\sqrt{\omega_{d}}}, \quad \int_{S^{d}} Y_{n k}(t) d s(t)=0
$$

для всех $n=1,2, \ldots, k=1, \ldots, \delta_{n}^{k}$, используя (1), получаем

$\int_{S^{d}} \sum_{n \in \mathbb{Z}_{+}} h_{j-1}(n) \frac{(2 n+d-1)}{\omega_{d}(d-1)} G_{n}^{((d-1) / 2)}(y \cdot t) d s(t)=\int_{S^{d}} h_{j-1}(0) Y_{01}(y) Y_{01}(t) d s(t)=1$.

Это влечет

$$
\left\|\Lambda_{j, \varnothing}\right\| \leqslant 1 .
$$

Аналогично, принимая во внимание, что $a_{\ell}^{(j)} \geqslant 0$, имеем

$$
\begin{aligned}
& \left|\sum_{\ell \in \omega} a_{\ell}^{(j+1)}\left\langle F, \widetilde{\Psi}_{j \ell}\right\rangle \Psi_{j \ell}(x)\right| \\
& \leqslant \sum_{\ell \in \omega} a_{\ell}^{(j+1)} \int_{S^{d}}|F(t)| \sum_{s=j-1}^{j} \sum_{n \in \mathbb{Z}_{+}} h_{s}(n) \frac{(2 k+d-1)}{\omega_{d}(d-1)} G_{n}^{((d-1) / 2)}\left(t \cdot \eta_{\ell}^{(j+1)}\right) d s(t) \\
& \quad \times \sum_{r=j-1}^{j} \sum_{k \in \mathbb{Z}_{+}} h_{r}(k) \frac{(2 n+d-1)}{\omega_{d}(d-1)} G_{k}^{((d-1) / 2)}\left(x \cdot \eta_{\ell}^{(j+1)}\right) \\
& \leqslant\|F\|_{\infty} \sum_{\ell \in \Omega_{j+1}} a_{\ell}^{(j+1)} \int_{S^{d}} \sum_{s=j-1}^{j} \sum_{n \in \mathbb{Z}_{+}} h_{s}(n) \frac{(2 n+d-1)}{\omega_{d}(d-1)} G_{n}^{((d-1) / 2)}\left(t \cdot \eta_{\ell}^{(j+1)}\right) d s(t) \\
& \quad \times \sum_{r=j-1}^{j} \sum_{k \in \mathbb{Z}_{+}} h_{r}(k) \frac{(2 n+d-1)}{\omega_{d}(d-1)} G_{k}^{((d-1) / 2)}\left(x \cdot \eta_{\ell}^{(j+1)}\right)
\end{aligned}
$$




$$
\begin{aligned}
=\|F\|_{\infty} \int_{S^{d}} d s(\eta) \int_{S^{d}} d s(t) \sum_{s=j-1}^{j} \sum_{n \in \mathbb{Z}_{+}} h_{s}(n) \sum_{\nu=1}^{\delta_{n}^{d}} Y_{n \nu}(\eta) Y_{n \nu}(t) \\
\quad \times \sum_{r=j-1}^{j} \sum_{k \in \mathbb{Z}_{+}} h_{r}(k) \sum_{\kappa=1}^{\delta_{k}^{d}} Y_{k \kappa}(\eta) Y_{k \kappa}(x)=4\|F\|_{\infty} .
\end{aligned}
$$

Отсюда и из $(9)$ следует $\left\|\Lambda_{j, \omega}\right\| \leqslant 5$. Теперь в силу теоремы Банаха-Штейнгауза достаточно проверить, что (7) вьполнено на множестве сферических полиномов. Пусть $F=\sum_{n=0}^{N} \sum_{\nu=1}^{\delta_{n}^{d}} \alpha_{n \nu} Y_{n \nu}$. Ввиду ортонормированности системы $\left\{Y_{n k}\right\}_{n, k}$ из соотношений (4) и (5) следует, что

$$
\Lambda_{j, \varnothing}(F)=\sum_{n=0}^{N} h_{j-1}^{2}(n) \sum_{\nu=1}^{\delta_{n}^{d}} \alpha_{n \nu} Y_{n \nu}
$$

Поскольку

$$
\lim _{j \rightarrow \infty} h_{j}(n)=1
$$

для любого фиксированного $n$, мы получаем (7) для $\omega=\varnothing$. Снова ввиду ортонормированности системы $\left\{Y_{n k}\right\}_{n, k}$ и (1) получаем

$$
\begin{aligned}
& \left|\sum_{\ell \in \omega} a_{\ell}^{(j+1)}\left\langle F, \widetilde{\Psi}_{j \ell}\right\rangle \Psi_{j \ell}(x)\right| \leqslant\left|\sum_{\ell \in \omega} a_{\ell}^{(j+1)} \sum_{n=0}^{N} \widetilde{g}_{i}(n) \sum_{\nu=1}^{\delta_{n}^{d}} \alpha_{n \nu} Y_{n \nu}\left(\eta_{\ell}^{(j+1)}\right) \Psi_{j \ell}(x)\right| \\
& \leqslant \max _{0 \leqslant n \leqslant N}\left|h_{j}(n)-h_{j-1}(n)\right| \sum_{\nu=1}^{\delta_{n}^{d}}\left|\alpha_{n \nu}\right|\left\|Y_{n \nu}\right\|_{\infty} \sum_{\ell \in \omega}\left|a_{\ell}^{(j+1)} \Psi_{j \ell}(x)\right| .
\end{aligned}
$$

На основании (2) и (1), принимая во внимание неотрицательность числа $a_{\ell}^{(j)}$ и $(3)$, имеем

$$
\begin{aligned}
& \sum_{\ell \in \omega}\left|a_{\ell}^{(j+1)} \Psi_{j \ell}(x)\right| \leqslant \sum_{\ell \in \Omega_{j+1}} a_{\ell}^{(j+1)}\left(\sum_{n \in \mathbb{Z}_{+}} h_{j}(n) \frac{(2 k+d-1)}{\omega_{d}(d-1)} G_{n}^{((d-1) / 2)}\left(t \cdot \eta_{\ell}^{(j+1)}\right)\right. \\
& \left.\quad+\sum_{k \in \mathbb{Z}_{+}} h_{j-1}(k) \frac{(2 k+d-1)}{\omega_{d}(d-1)} G_{k}^{((d-1) / 2)}\left(t \cdot \eta_{\ell}^{(j+1)}\right)\right) \\
& =\int_{S^{d}} d s(\eta)\left(\sum_{n \in \mathbb{Z}_{+}} h_{j}(n) \sum_{\nu=1}^{\delta_{n}^{d}} Y_{n \nu}(\eta) Y_{n \nu}(t)+\sum_{k \in \mathbb{Z}_{+}} h_{j-1}(k) \sum_{\kappa=1}^{\delta_{n}^{d}} Y_{k \kappa}(\eta) Y_{k \kappa}(t)\right)=2 .
\end{aligned}
$$

Сопоставляя это соотношение с (10) и (11), получаем

$$
\lim _{j \rightarrow \infty} \max _{\omega \subset \Omega_{j+1}}\left\|\sum_{\ell \in \omega} a_{\ell}^{(j+1)}\left\langle F, \widetilde{\Psi}_{j \ell}\right\rangle \Psi_{j \ell}\right\|_{\infty}=0
$$

что и оставалось проверить. 
4. В этом пункте мы построим полиномиальный базис в $C\left(S^{d}\right)$. Будем использовать подход, основанньй на теоремах Пэли-Винера и Крейна-Мильмана-Рутмана (см. [1]). Пусть $\left\{Q_{k}\right\}_{k=0}^{\infty}-$ базис в банаховом пространстве $H$, и пусть $\alpha_{k} \in H^{*}, k=0,1, \ldots,-$ функционалы, определяюшие коэффициенты разложения по этому базису. Если $P_{k} \in H$ и

$$
\left\|P_{k}-Q_{k}\right\| \leqslant \frac{2^{-k-2}}{\left\|\alpha_{k}\right\|}=: \lambda_{k}
$$

для всех $k=0,1, \ldots$, то последовательность $\left\{P_{k}\right\}_{k=0}^{\infty}$ является базисом в $H$. Мы начнем с нахождения отправного базиса $\left\{Q_{k}\right\}$ в $C\left(S^{d}\right)^{1}$.

Шаг 1. Построение базиса в пространствах $C\left([0,1]^{d}\right)$ и

$$
C_{0}\left([0,1]^{d}\right):=\left\{f \in C\left([0,1]^{d}\right): f(x)=0 \forall x \in \partial\left(\left([0,1]^{d}\right)\right\} .\right.
$$

Пусть $\left\{f_{n}\right\}_{n=0}^{\infty}-$ базис Фабера-Шаудера (см., например, [14, гл. 6]) в $C[0,1]$, определяемьй формулами: $f_{0}(x)=1, f_{1}(x)=x, x \in[0,1]$; для $n=2^{k}+i, k=0,1,2, \ldots$, $i=1,2, \ldots, 2^{k}$, функции $f_{n}$

$$
\begin{gathered}
\text { линейны и непрерьвны на }\left[\frac{i-1}{2^{k}}, \frac{2 i-1}{2^{k+1}}\right] \text { и на }\left[\frac{2 i-1}{2^{k+1}}, \frac{i}{2^{k}}\right], \\
f_{n}(x)=\left\{\begin{array}{l}
0, \text { если } x \notin\left(\frac{i-1}{2^{k}}, \frac{i}{2^{k}}\right), \\
1, \text { если } x=\left(\frac{2 i-1}{2^{k+1}}\right) .
\end{array}\right.
\end{gathered}
$$

Тензорное произведение $d$ систем $\left\{f_{n}\right\}$ (должньм образом занумерованное) является базисом в $C\left([0,1]^{d}\right)$ (см., например, $[14$, гл. $\left.1, \S 18]\right)$. Обозначим этот базис через $\mathscr{B}=$ $\left\{F_{k}\right\}_{k=1}^{\infty}$. Элементы множества $\mathscr{B}$ - функции вида

$$
F(x)=f_{n_{1}}\left(x_{1}\right) f_{n_{2}}\left(x_{2}\right) \cdots f_{n_{d}}\left(x_{d}\right), \quad x_{j} \in[0,1], \quad n_{j} \in \mathbb{Z}_{+}, \quad j=0,1, \ldots, d .
$$

Обозначим через $\mathscr{B}_{0}$ подмножество множества $\mathscr{B}$, которое состоит из всех таких функций $F$, что $n_{j} \neq 0, n_{j} \neq 1, j=0,1, \ldots, d$ (занумерованных в том же порядке). Нетрудно проверить, что $\mathscr{B}_{0}=\left\{F_{k}^{(0)}\right\}_{k=1}^{\infty}-$ базис в $C_{0}\left([0,1]^{d}\right)$.

Шаг 2. Построение базисов в пространствах $C\left(B^{d}\right)$ и

$$
C_{0}\left(B^{d}\right):=\left\{f \in C\left(B^{d}\right): f(x)=0 \forall x \in \partial B^{d}\right\} .
$$

$\mathrm{C}$ помощью замены переменных мы можем заменить $[0,1]^{d}$ на $[-1,1]^{d}$, сохранив те же обозначения $\mathscr{B}, \mathscr{B}_{0}$ для соответствуюших базисов. Зададим отображение $\varphi: B^{d} \rightarrow$ $[-1,1]^{d}$ по формулам: если $x=(\rho, \theta), 0 \leqslant \rho \leqslant 1, \theta \in S^{d-1}$, то $\varphi(x)=(\rho r(\theta), \theta)$, где $r(\theta)$ - длина отрезка $\left\{x=(t, \theta): t \geqslant 0, x \in[-1,1]^{d}\right\}$. Ясно, что $\varphi$ взаимно однозначно отображает $B^{d}$ на $[-1,1]^{d}$ и функции $G_{k}:=F_{k}(\varphi), k=1,2, \ldots$, образуют базис в $C\left(B^{d}\right)$. Обозначим этот базис через $\mathscr{B}^{\prime}$. Аналогично из $\mathscr{B}_{0}$ строится базис $\mathscr{B}_{0}^{\prime}=\left\{G_{k}^{(0)}\right\}_{k=1}^{\infty}$ в $C_{0}\left(B^{d}\right)$.

\footnotetext{
${ }^{1}$ Вероятно конкретные базисы в $C\left(S^{d}\right)$ известны. Поскольку нам не удалось что-либо найти, приводим свою конструкцию.
} 
Шаг 3. Построение базиса в $C\left(S^{d}\right)$. Пусть $C^{(e)}\left(S^{d}\right)$ и $C^{(o)}\left(S^{d}\right)-$ соответственно множества функций $f \in C\left(S^{d}\right)$ таких, что

$$
f\left(x_{1}, x_{2}, \ldots, x_{d}, x_{d+1}\right)=f\left(x_{1}, x_{2}, \ldots, x_{d},-x_{d+1}\right)
$$

и

$$
f\left(x_{1}, x_{2}, \ldots, x_{d}, x_{d+1}\right)=-f\left(x_{1}, x_{2}, \ldots, x_{d},-x_{d+1}\right) .
$$

Каждое $f \in C\left(S^{d}\right)$ представимо в виде

$$
f=f^{(e)}+f^{(o)}
$$

где $f^{(e)} \in C^{(e)}\left(S^{d}\right), f^{(o)} \in C^{(o)}\left(S^{d}\right)$. Очевидно, что если $\left\{H_{k}^{(e)}\right\}_{k=1}^{\infty}$ и $\left\{H_{k}^{(o)}\right\}_{k=1}^{\infty}-$ базисы в $C^{(e)}\left(S^{d}\right)$ и $C^{(o)}\left(S^{d}\right)$ соответственно, то система $\left\{H_{k}^{(e)}, H_{k}^{(o)}\right\}_{k=1}^{\infty}-$ базис в $C\left(S^{d}\right)$. Таким образом осталось найти базисы в $C^{(e)}\left(S^{d}\right)$ и $C^{(o)}\left(S^{d}\right)$. Для $x \in S^{d}$ положим

$$
\begin{aligned}
& H_{k}^{(e)}(x):=G_{k}\left(x_{1}, \ldots, x_{d}\right), \\
& H_{k}^{(o)}(x):= \begin{cases}G_{k}^{(0)}\left(x_{1}, \ldots, x_{d}\right), & \text { если } x_{d+1} \geqslant 0, \\
-G_{k}^{(0)}\left(x_{1}, \ldots, x_{d}\right), & \text { если } x_{d+1} \leqslant 0 .\end{cases}
\end{aligned}
$$

Ясно, что системы $\left\{H_{k}^{(e)}\right\}_{k=1}^{\infty}$ и $\left\{H_{k}^{(o)}\right\}_{k=1}^{\infty}$ являются требуемыми базисами.

Переобозначим систему $\left\{H_{k}^{(e)}, H_{k}^{(o)}\right\}_{k=1}^{\infty}$ через $\left\{Q_{k}\right\}_{k=1}^{\infty}$, и пусть $\left\{\alpha_{k}\right\}_{k=1}^{\infty}$ - последовательность соответствующих функционалов, определяющих коэффициенты разложений, $\lambda_{k}=2^{-k-2} /\left\|\alpha_{k}\right\|$. Для заданной последовательности натуральных чисел $\left\{N_{k}\right\}$ положим $P_{k}:=\sigma_{N_{k}}^{\beta}\left(Q_{k}\right)$, где $\sigma_{m}^{\beta}-(C, \beta)$-средние ряда Фурье по сферическим гармоникам, $\beta>(d-1) / 2$ (см., например, $[15, \S 2.3])$. Поскольку $\left\{\sigma_{m}^{\beta}(f)\right\}_{m=1}^{\infty}$ равномерно сходятся к $f \in C\left(S^{d}\right)$, мы можем выбрать $\left\{N_{k}\right\}$ так, чтобы $\left\|Q_{k}-P_{k}\right\| \leqslant \lambda_{k}$. Таким образом, $\left\{P_{k}\right\}_{k=1}^{\infty}-$ полиномиальный базис в $C\left(S^{d}\right)$.

\section{СПИСОК ЦИТИРОВАННОЙ ЛИТЕРАТУРЫ}

[1] Foias C., Singer I. Some remarks on strongly independent sequences and bases in Banach spaces // Rev. Math. Pure et Appl. Acad. R. P. R. 1961. V. 6. № 3. P. 589-594.

[2] Привалов Ал. А. О росте степеней полиномиальных базисов и приближении тригонометрических проектовов // Матем. заметки. 1987. Т. 42. № 2. С. 207-214.

[3] Lorentz R. A., Sahakian A. A. Orthogonal trigonometric Schauder bases of optimal degree for $C(0,2 \pi) / /$ J. Fourier Anal. Appl. 1994. V. 1. № 1. P. 103-112.

[4] Скопина М. А. Ортогональные полиномиальные базисы Шаудера $C[-1,1]$ с оптимальным ростом степеней // Матем. сб. 2001. Т. 192. № 3. С. 115-136.

[5] Freeden W., Schreiner M. Orthogonal and non-orthogonal multiresolution analysis, scale discrete and exact fully discrete wavelet transform on the sphere // Constructive Approximation. 1998. V. 14. P. 493-515.

[6] Farkov Yu. B-spline wavelets on the sphere // "Self-Similar Systems", Proceedings of the International Workshop (July 30-August 7, 1998, Dubna, Russia). Dubna: JINR, E5-99-38, 1999. P. 79-82. 
[7] Skopina M. Polynomial expansions of continuous functions on the sphere and on the disk // Preprint № 2001:5. University of South Carolina, Department of Mathematics, 2001.

[8] Müller C. Spherical Harmonics. Berlin: Springer-Verlag, 1966. (Lecture Notes in Math. V. 17.)

[9] Стейн И., Вейс Г. Введение в гармонический анализ на евклидовых пространствах. М.: Мир, 1974.

[10] Mhaskar H. N., Narcowich F. J., Ward J. D. Spherical Marcinkievicz-Zygmund inequalities and positive quadrature // Math. Comp. 2001. V. 70. № 235. P. 1113-1130.

[11] Mhaskar H. N., Narcowich F. J., Ward J.D., Prestin J. Polynomial frames on the sphere // Adv. Comput. Math. 2000. V. 13. P. 387-403.

[12] Askey R. Orthogonal polynomials and spherical functions. Philadelphia: SIAM, 1975.

[13] Сегё Г. Ортогональные многочлены. М.: Физматгиз, 1962.

[14] Кашин Б. С., Саакян А. А. Ортогональные ряды. М.: АФЦ, 1999.

[15] Wang Kunyang, Li Luoking. Harmonic analysis and approximation on the unit sphere. Graduate Series in Mathematics, 2000.

(А. Аскари-Хеммат, М. А. Дегхан) Университет Валиаср, Иран

Поступило

(М. А. Скопина) С.-Петербургский государственный университет

08.05 .2002

E-mail: (А. Аскари-Хеммат) asat@yahoo.com

(M. А. Дегхан) asat@arg3.uk.ac.ir

(M. А. Скопина) skopina@sk.usr.lgu.spb.su 